\title{
CIRED 2017: Bericht über Session 2 - Spannungsqualität und elektromagnetische Verträglichkeit
}

\author{
H. Renner OVE \\ Online publiziert am 22. November 2017 \\ (C) Springer-Verlag GmbH Austria, ein Teil von Springer Nature 2017
}

Wie auch in den vergangenen Jahren werden in Session 2 die Themen „Power Quality" und „Elektromagnetische Phänomene" behandelt. Unter Leitung des bewährten Teams - Herwig Renner (TU Graz) als Chairman sowie Britta Heimbach (EW Zürich) und Jan Desmet (Universität Gent) als Special Reporters - wurde in der Konferenz ein ausgezeichneter Überblick aus Sicht der Netzbetreiber, Kunden, Anlagenhersteller, Regulatoren und Forschungseinrichtungen gegeben. Session 2 gliederte sich in Glasgow in die folgenden Themenblöcke:

- Block 1: Elektrische und magnetische Felder, Sicherheit und Beeinflussungen

- Block 2: Spannungsqualität in Hinblick auf dezentrale Einspeisung und Elektromobilität

- Block 3: Messung, Analyse und Maßnahmen zur Verbesserung der Spannungsqualität

- Block 4: Power Quality Monitoring, Datenmanagement und regulatorische Belange

Insgesamt wurden 107 Beiträge nach sorgfältiger Begutachtung angenommen und in der Main-Session 22 Beiträge präsentiert. Österreich war mit einem Beitrag in Block 2 vertreten.

In Block 1 wurde die Einführung des Risikobegriffs im Zusammenhang mit Erdungen elektrischer Anlagen diskutiert. Das Thema wird derzeit von der CIGRE/CIRED-Arbeitsgruppe B3.35 (Co-Chair Prof. Fickert, TU Graz) untersucht. Ziel ist es, diese Aspekte in der internationalen Normung für Erdungen zu implementieren.

Im Bereich der elektromagnetischen Beeinflussung wurde die Auswirkung von hochfrequenten Oberschwingungen (Supra-Harmonics), wie sie beispielsweise von PV-Wechselrichtern emittierte werden, auf die Zuverlässigkeit von Powerline Communication (PLC) diskutiert. Es wurde übereinstimmend festgestellt, dass aus heutiger Sicht keine Probleme durch hochfrequente elektrische oder magnetische Felder zu erwarten sind. Im österreichischen Beitrag (Abart e.a.) wurde der umgekehrte Effekt, die Emission hochfrequenter Felder durch PLC-Systeme (wie sie z. B. in der Smart-Meter-Kommunikation eingesetzt werden), untersucht. Die Autoren kommen zum Schluss, dass diese Emission kein gesundheitliches Risiko darstellt.

Ein weiterer Schwerpunkt war die Schirmung magnetischer 50$\mathrm{Hz}$-Felder durch Composite-Elemente, die mit sandwichartigem Aufbau elektrisch leitfähige und ferromagnetische Materialien kombinieren und damit eine verbesserte Schirmwirkung erzielen.

In Block 2 wurde der Einfluss von dezentraler Erzeugung und Elektromobilität auf die Spannungsqualität diskutiert. Mehrfach wurde auf den wesentlichen Einfluss der Netzimpedanz und der Oberschwingungsvorbelastung auf die Oberschwingungsemission hingewiesen. Insbesondere bei Umrichter-gekoppelter Einspeisung sind die Phänomene der sekundären Emission und der Auslöschung von Oberschwingungen ein Thema. Darunter ist zu verstehen, dass die Oberschwingungsemission nicht als konstanter, eingeprägter Strom zu sehen ist, sondern stark von den Umgebungsbedingungen abhängt. Dies muss auch bei entsprechenden Simulationsrechnungen berücksichtigt werden. Ein weiteres interessantes Thema ist die Reglerinstabilität im Zusammenhang mit schwachen Netzen oder Netzresonanzen. Diese werden unter anderem im hochfrequenten Bereich durch EMV-Filter von Umrichtern beeinflusst. Dieses Thema wurde auch in Block 3 aufgegriffen. Für den Bereich PV wurde das Problem der Spannungsunsymmetrie durch einphasig angeschlossene PV-Anlagen diskutiert. Es wurden Ergebnisse von umfangreichen Simulationsrechnungen präsentiert und gezeigt, dass für eine große Anzahl zufällig angeschlossener PV-Anlagen in seltenen Fällen die Spannungsunsymmetrie einen Wert von $2 \%$ überschreiten kann. In einer Round-Table-Diskussion wurden die ersten Ergebnisse der CIGRE-Arbeitsgruppe C4/C6.29 "Power quality aspects of solar power" vorgestellt. Nicht überraschend wurde die Gefahr von unzulässigen Spannungsanhebungen als wesentliches Problem hervorgehoben.

Ein Beitrag über die synchronisierte Messung von schnellen Spannungsänderungen mit dem Ziel, daraus die Flickeremission von Kundenanlagen ableiten zu können, löste eine generelle Diskussion über die Zukunft des Qualitätsparameters "Flicker" aus. Es ist davon auszugehen, dass zukünfting Anforderungen an die Flickerimmunität von Beleuchtungsmitteln normativ festgeschrieben wird.

Spannungseinbrüche (Dips) sind nach wie vor ein wesentliches Problem für Kunden. Nach und nach werden nationale Standards mit Grenzwerten für die zulässige Anzahl an Dips (klassiert nach Restspannung und Dauer) implementiert. Diese basieren oft auf dem von CIGRE/CIRED eingeführten Konzept der geteilten Verantwortung von Netznutzer und Netzbetreiber. Eine interessante Methode zur Fehlerortbestimmung, beruhend auf synchronisierter Messung von Dips, wurde ebenfalls in Block 4 vorgestellt.

Im Bereich des Spannungsqualitäts-Monitorings hat sich PQDIF (Power Quality Data Interchange Format) mehr oder weniger als Standardformat etabliert. Die dazugehörige IEEE-Norm 1159.3 wird derzeit überarbeitet.

Eine umfassende Zusammenfassung der eingereichten Beiträge in Session 2 ist wie immer im "Special Report" zu finden, der auf der Konferenzhomepage http://www.cired-2017.org/ zur Verfügung gestellt wird

24th International Conference on Electricity Distribution, Glasgow, Schottland, 12. bis 15. Juni 2017.

Renner, Herwig, Institut für Elektrische Anlagen, Technische Universität Graz, Inffeldgasse 18/l, 8010 Graz, Österreich (E-Mail: herwig.renner@tugraz.at) 